\title{
Research on Cognitive Load Theory and its Design Implications for E-Learning
}

Jeroen J. G. van Merriënboer

Educational Technology Expertise Center, Open University of the Netherlands

\author{
Paul Ayres
}

School of Education, University of New South Wales, Australia

\begin{abstract}
This introduction to the special issue provides a context for the contributing articles. For readers who are not familiar with Cognitive Load Theory (CLT), it provides a very brief description of assumptions regarding memory systems and learning processes, different types of cognitive load (intrinsic, extraneous, and germane), and design implications. Whereas traditional CLT research focused on instructional methods to decrease extraneous cognitive load that is not directly relevant for learning, contributions to this special issue represent wider perspectives that reflect new developments in CLT. These papers have been organized into three categories: (1) methods to decrease intrinsic cognitive load and deal with high-element interactivity materials, (2) methods to increase germane cognitive load that is directly relevant for learning, and (3) methods to deal with differences in learner's individual levels of expertise and expertise development. To conclude, design implications for (adaptive) e-learning are discussed.
\end{abstract}

Educational Technology, Research \& Development, 53(3), 5-13. 